\title{
Double Heterozygous Mutations in the BRCA2 and ATM Genes: A Case Report and Review of the Literature
}

\author{
Neslihan Duzkale Teker ${ }^{\mathrm{a}} \quad$ Nilnur Eyerci $^{\mathrm{b}}$ \\ ${ }^{a}$ Department of Medical Genetics, Diskapi Yildirim Beyazit Training and Research Hospital, Ankara, Turkey; \\ ${ }^{b}$ Department of Medical Biology, Faculty of Medicine, Kafkas University, Kars, Turkey
}

\section{Established Facts}

- DNA repair genes, such as BRCA1/2 and $A T M$, are associated with cancer development.

\section{Novel Insights}

- In this patient, we detected pathogenic variants of the BRCA2 and ATM genes for the first time as double heterozygous. These two genes may have contributed synergistically to the multiple primary malignant neoplasia phenotype.

\section{Keywords}

BRCA · ATM - Breast cancer · Pancreas cancer · Multiple primary malignant neoplasia

\section{Abstract}

Introduction: Germline mutations of the BRCA1 and BRCA2 genes are responsible for about a quarter of hereditary breast cancers (BCs). In this study, we aimed to determine the importance of rare double heterozygous $(\mathrm{DH})$ pathogenic variant carriership in BRCA2 and ATM genes in a patient diagnosed with $B C$ and pancreas cancer (PC). Case Report: A 54-year-old female patient was diagnosed with $\mathrm{BC}$ at the age of 34 years and with PC at the age of 48 years. The multigene panel and next-generation sequencing technique were used to evaluate the status of the patient's cancer susceptibility genes. Pathogenic variants c.537dup (p. Ile180Tyrfs*3) in the BRCA2 gene and c.5065C > T (p.GIn1689Ter) in the ATM gene were detected as $\mathrm{DH}$ in the patient. Co-
}

segregation analysis was performed on the relatives of the patient using Sanger sequencing. Discussion/Conclusion: Multiple primary malignant neoplasms can be encountered more frequently in $\mathrm{DH}$ pathogenic variant carriers, and the diagnosis of malignancies can be made at an earlier age through surveillance guided by genetic testing. In this rare case, more patient studies are needed to determine the contribution of DH in BRCA2 and ATM genes to the phenotype.

(c) 2020 S. Karger AG, Basel

\section{Introduction}

About $5-10 \%$ of all breast cancers (BCs) are hereditary, and about $25 \%$ of hereditary ones are associated with the BRCA1 and BRCA2 genes, which play an important role in the DNA repair mechanism [1]. Genes such as ATM, CHEK2, PALB2, PTEN, STK11, TP53, CDH1 and $N F 1$, which are involved in maintenance of genomic in- 


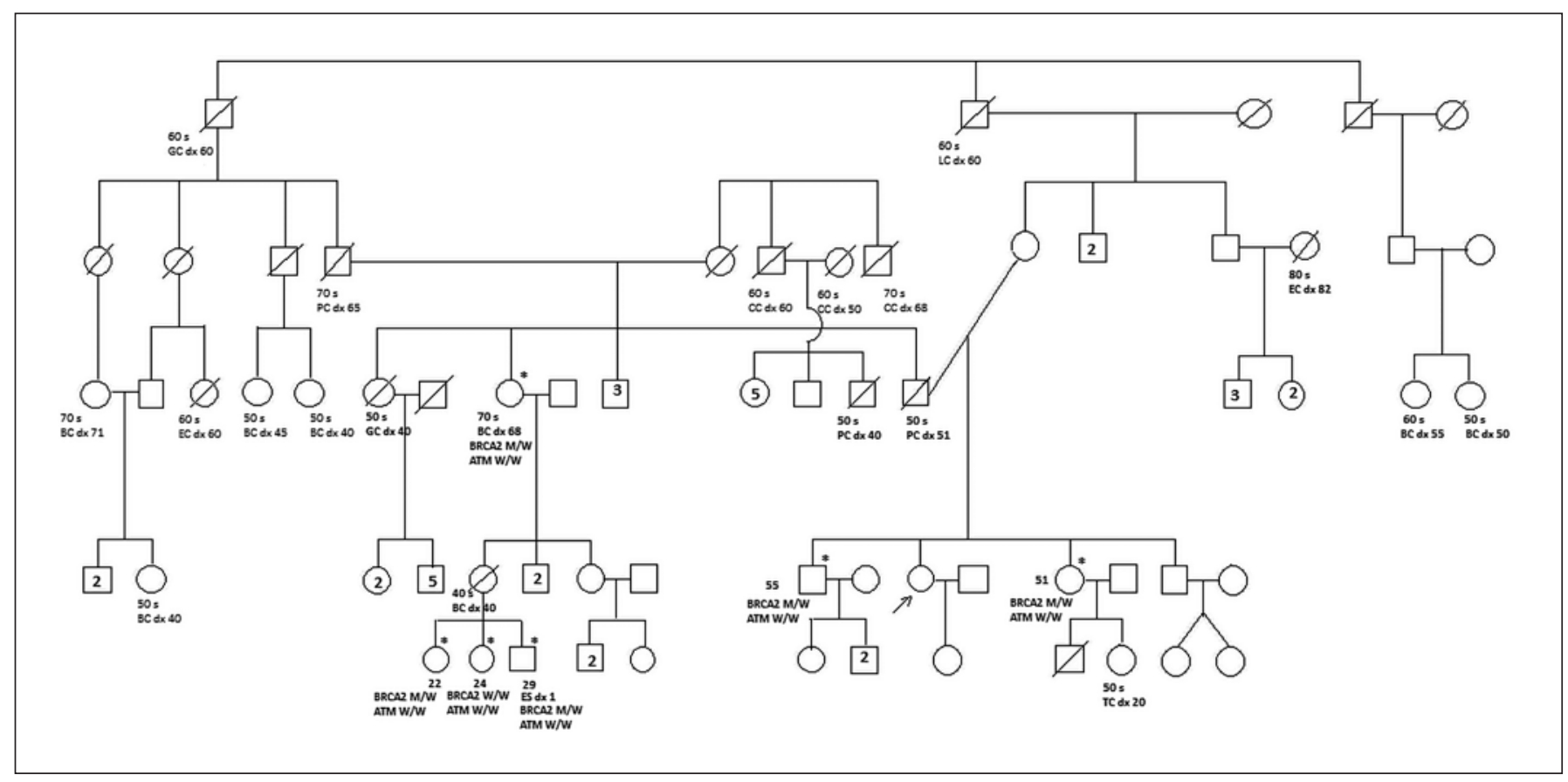

Fig. 1. Family pedigree. The proband is indicated with an arrow. The ages and phenotypes of family members are listed under the symbols. Individuals undergoing genetic analysis are indicated by asterisks. BC, breast cancer; PC, prostate cancer; TC, thyroid cancer; GC, gastric cancer; EC, endometrium cancer; LC, lung cancer; CC, colon cancer; ES, Ewing sarcoma; dx, diagnosed; M/W, heterozygous genotype; W/W, wild genotype.

tegrity, also cause inherited BC syndromes [2]. Detection of germline mutations of these genes with NGS (nextgeneration sequencing) method allows development of familial screening, treatment and prevention strategies in carriers [3].

To date, there has not been a large number of published case reports or patient series referring to the coexistence of two pathogenic variants and multiple types of cancer in BC-associated genes. Double heterozygous $(\mathrm{DH})$ cases have been described by the presence of pathogenic variants in BRCA1, BRCA2, PALB2, CHEK2, BLM or NBN $[4,5]$.

In this study, we report the genotype-phenotype correlation of a woman with a pathogenic variant in $B R C A 2$ and ATM genes.

\section{Case Report}

The 53-year-old female, who had no prior history of chronic disease other than allergic rhinitis, was diagnosed with $\mathrm{BC}$ when she was 34 years old. The patient underwent a left breast modified radical mastectomy and axillary lymphadenectomy. Pathological evaluation of the tumoral tissue indicated that it was an invasive mixed (ductal and lobular) carcinoma, and in the immunohistochemical examination, estrogen and progesterone receptors were strongly positive. Medical treatment of the patient consisted of six cycles of FEC (5-fluorouracil $500 \mathrm{mg} / \mathrm{m}^{2}$, epirubicin $60 \mathrm{mg} / \mathrm{m}^{2}$, and cyclophosphamide $500 \mathrm{mg} / \mathrm{m}^{2}$ ), followed by tamoxifen for 5 years.
Fourteen years after BC, a mass was noticed in the patient's pancreatic head during routine controls. A diagnosis of moderate differential ductal adenocarcinoma was made in the pathological evaluation of this mass, which was removed with Whipple operation. In the literature, the combined adjuvant treatment program has been reported to be highly tolerable and effective in PC [6]. As such, medical therapy management was planned as adjuvant $4 \mathrm{cy}-$ cles of gemcitabine chemotherapy $\left(1,500 \mathrm{mg} / \mathrm{m}^{2}\right)$, followed by simultaneous radiotherapy and 5 cycles of capecitabine chemotherapy $\left(1,330 \mathrm{mg} / \mathrm{m}^{2}\right)$. The preferred adjuvant radiotherapy dose in this patient was $180 \mathrm{cGy}$ daily in 28 fractions by the IMRT technique. The patient's TNM classification for both BC and PC was determined as T2N0M0.

Analysis of the patient's family tree revealed a considerable number of relatives diagnosed with cancer. The pathogenic BRCA2 c.537dup (p.Ile180Tyrfs*3) variant was found in one sister of the patient, one brother and one aunt, and in two grandchildren of this aunt. In the patient's aunt BC was diagnosed, and 1 grandson of this aunt was diagnosed with Ewing sarcoma. Other relatives who were carriers of this pathogenic variant were healthy. The pathogenic variant in the ATM gene, c.5065C > T (p.Gln1689Ter), could not be detected in any of the tested relatives (Fig. 1). The patient's daughter and mother refused to undergo a genetic test. It was not possible to do the study with other relatives because they were either not alive or the patient was unable to contact them.

\section{Method}

This study was conducted at the Abdurrahman Yurtaslan Oncology Hospital, Department of Medical Genetics, Ankara, Turkey. Genetic analyses of patient and her family members were performed by the NGS method. DNA was extracted from peripheral blood. The exonic and flanking intronic regions and copy number variations (CNVs) of 26 genes analyzed by NGS include ATM, 
BARD1, BLM, BRCA1, BRCA2, BRIP1, CDH1, CHEK2, EPCAM, FAM175A, MEN1, MLH1, MRE11A, MSH2, MSH6, MUTYH, NBN, PALB2, PMS2, PTEN, RAD50, RAD51C, RAD51D, STK11, TP53 and XRCC2.

Analysis of the variants uncovered pathogenic variants in two genes: NM_000059.3 (BRCA2):c.537dup (p.Ile180Tyrfs*3) and NM_000051.3 (ATM): c.5065C > T (p.Gln1689Ter), both were detected in the heterozygous state. The BRCA2 variant is located in exon 7 (of 24 exons total), it duplicates a single base pair (T) which leads to the formation of frameshift and a premature stop codon. This is predicted to result in either truncation or nonsense mediated mRNA decay (NMD). The ATM variant is located in exon 34 (of 63 exons total) and directly creates a premature stop codon. The premature translational stop signal generated by both of these variants is expected to result in either a missing protein product or truncated protein with impaired function. These variants were not observed in healthy controls in population databases. In recent years, the ATM variant in a patient with ovarian cancer [3] and BRCA2 variant in a patient with $\mathrm{BC}$ has been reported in the literature [7]. Studies have notified that loss-of-function variants in these genes are pathogenic [8-10].

\section{Discussion}

Advances in NGS technology and making this technology more accessible have provided clinicians with a great advantage in many areas of medicine. In addition, genotype-phenotype correlation has become more widely defined in patients thanks to multi-gene disease panels. Considering the criteria in the NCCN guideline, certain patients diagnosed with $\mathrm{BC}$ need a BRCA1/2 testing [11]. However, in individuals with multiple primary malignant neoplasms (MPMN), more comprehensive data can be easily obtained with multi-gene panels. Although there are no clear guidelines regarding the patient selection criteria to be used in the multigene test panel, in this study, we analyzed our patient with an NGS panel since she was diagnosed with MPMN.

As a result of the analysis, the patient was diagnosed with $B R C A 1$ and BRCA2 associated hereditary breast and ovarian cancer syndrome (HBOC) because she had a pathogenic variant in the BRCA2 gene. Studies have shown that the lifetime risk of $\mathrm{BC}$ is $46-87 \%$ in $B R C A 1$ mutation carriers and $38-84 \%$ in $B R C A 2$ mutation carriers. The second primary $\mathrm{BC}$ risk until 70 years of age in female $\mathrm{HBOC}$ patients with $\mathrm{BC}$ is $83 \%$ in $B R C A 1$ mutation carriers and $62 \%$ in $B R C A 2$ mutation carriers. Also, the risk of developing ovarian cancer up to 70 years is $39-63 \%$ in $B R C A 1$ mutation carriers and $16.5-27 \%$ in BRCA2 mutation carriers [12].

In addition to the significantly increased risk of breast and ovarian cancers in BRCA1/2 mutation carriers, an increased risk has also been reported in many organ cancers such as pancreas, prostate, skin, stomach, colon, buccal cavity, pharyngeal, kidney, bile duct, cervical, endometrium, bone and gallbladder $[13,14]$.
Due to the high penetration of $B R C A 1 / 2$ genes, the option of prophylactic bilateral mastectomy and prophylactic oophorectomy can be offered to the patient in order to prevent the expected primary symptoms in female HBOC cases. In these women, $\mathrm{BC}$ screening in general is planned as a combination of monthly breast self-examination as well as an annual or 6-month breast examination, annual mammography and breast MRI by a clinician. Although not effective in detecting early-stage ovarian cancer in high-risk or average-risk women, annual transvaginal ultrasound and CA-125 concentration levels starting from 35 years of age may be considered for screening ovarian cancer in $\mathrm{HBOC}$ cases. BC screening in male HBOC cases, breast self-examination training from the age of 35 years and annual breast examination by a clinician have been recommended. These patients also require annual prostate cancer screening from the age of 45 years. Although PC screening is not generally recommended for asymptomatic individuals, in both genders, melanoma screening should be individualized according to family history [12].

Comprehensive genetic counseling on HBOC was given to the patient and her relatives. In relatives who are carriers of the germline pathogenic variant in BRCA2 gene and who have not yet been diagnosed with cancer, it might be due to reduced penetration and variability in the age of disease onset. These individuals were also informed about the importance of certain environmental factors as well as individual differences in the carcinogenesis process. Determination of a pathogenic variant in $B R C A 2$ tumor suppressor gene in our patient and her healthy relatives has enabled these individuals to be included in an intensive cancer screening, prevention, and monitoring program.

In our patient, a second pathogenic variant was detected in the ATM gene. Playing an important role in the checkpoints of the cell cycle, the ATM gene provides the recognition and repair of double-strand DNA breaks. Pathogenic variants of this gene are observed in 1-2\% of the adult population in the heterozygous state. The risk for the development of any type of cancer in ATM heterozygous carriers is thought to be 2-6 times higher than in non-carriers [15]. The $\mathrm{BC}$ risks of these individuals are approximately 2-3 times higher compared to the general population. In terms of lifetime risk of $\mathrm{BC}$ compared to high penetration $B R C A 1 / 2$ genes, pathogenic variants of $A T M$ gene show more moderate penetration [16]. However, since the lifetime risk of $\mathrm{BC}$ in these women is over $25 \%$, screening with annual mammography and MRI should be planned, starting around the age of 40 years. If these individuals have a strong family history of $\mathrm{BC}$, the screening program should start at an earlier age. In order to avoid radiation exposure due to annual mammography, screening is performed only with MRI; however, a 
combination of mammography and MRI can be recommended to increase sensitivity in high-risk individuals. It has been shown that ATM heterozygous mutations increase cellular radiosensitivity by around 2.6 times [17] Although it has been demonstrated by in vitro studies, an increase in radiotherapy toxicity in the heterozygous carriers of pathogenic ATM variants in vivo are controversial [18-22].

It is considered that radiotherapy administered to this patient group does not trigger the formation of secondary cancer $[23,24]$. In some publications, it has been suggested that the systemic chemotherapy of these individuals provides an increased risk of moderate toxicity [25]. Among the reasons for the inconsistency in the results, insufficient number of patients studied, methodological differences, and changes in the expression of genes can be counted. In conclusion, it should be kept in mind that a wide variety of clinical responses can be obtained in the radiotherapy and chemotherapy strategy of $\mathrm{DH}$ individuals and the risk of toxicity may increase; therefore, attention should be paid to the dose adjustment of the adjuvant treatment.

Although there are no large-scale case series in the literature investigating individuals carrying pathogenic variants of cancer susceptibility genes as $\mathrm{DH}$, there are publications reporting that these individuals are diagnosed with cancer at an early age and that more than one primary tumor is more common in them [21, 26-28]. In a study comparing $\mathrm{DH}$ with single mutation carriers, it was shown that there was no significant difference for both groups in parameters such as age at diagnosis of malignancy, lifetime cumulative malignancy risk, and frequency of MPMN [4]. In another study, it was reported that the age of malignancy development and clinical course may vary in $\mathrm{DH}$ individuals from different ethnicities [28].

Until now, there are only few case reports in the literature in which various susceptibility genes carried DH. In one report, a female patient with double heterozygosity in BRCA1 and ATM genes followed with a diagnosis of MPMN (breast, pancreas and uterus) was presented. In this patient, early age of cancer onset and a severe clinical course caused by DH were not observed [29]. However, in BRCA1 knockout mice, it has been noticed that those with $A T M$ heterozygous mutation in combination have a more severe course of $\mathrm{BC}$, and it was thought that this situation may create a synergistic interaction in tumorigenesis [30].

Some in vitro studies have shown that DH in ATM and $B R C A 1$ genes can create a higher rate of cell transformation compared to single heterozygosity [31]. Although in vitro studies and animal studies help shape predictions about the clinical course of patients, reports on DH individuals are limited in the literature. In addition, data on many environmental and individual risk factors that may affect the age of diagnosis in this disease group, which might be extremely heterogeneous in different populations, have not been fully reported in the literature. The details of screening range and methods and treatment strategy (prophylactic treatment, chemotherapy and radiotherapy) in cancer cases are not complete. For all these reasons, a standard algorithm for the screening, treatment and follow-up of DH patients has still not been established.

Although DH pathogenic variants in BRCA1 and ATM have been previously reported in a case with MPMN [29], to the best of our knowledge, it is for the first time in the literature that pathogenic germline variants of $B R C A 2$ and $A T M$ genes in our patient presented in a DH state. Women who are carriers of pathogenic variants in the $B R C A 2$ gene are already expected to develop $\mathrm{BC}$ and PC at an early age. Heterozygous mutation carriers of the $A T M$ gene have been reported to have an increased risk, which is established in $\mathrm{BC}$, but this is contradictory in organ cancers such as pancreatic, ovarian, colorectal, thyroid, lung, prostate and stomach cancer $[32,33]$. The synergistic interaction between $B R C A 2$ and $A T M$ genes and $\mathrm{DH}$ variants may have contributed to our patient's diagnosis of early BC and MPMN.

At the present time, in the literature, there is still no clear information about the possible effects of $\mathrm{DH}$ in humans. It is necessary to examine more cases about which $\mathrm{DH}$ genes are found in the individual, the type of mutation in these genes, functionality of the gene product, and clinical details. In this way, in the near future, the mystery regarding the genotype-phenotype relationships in these individuals may be better elucidated.

\section{Acknowledgements}

The authors thank the patients who agreed to be included in this study.

\section{Statement of Ethics}

Before the study, written informed consent was obtained from the patient and her relatives who underwent genetic analysis for the publication of this case report and accompanying images. The independent Ethics Committee of the Abdurrahman Yurtaslan Oncology Hospital approved this descriptive case study (Document No.: 2020-02/536).

The present study involved human participants, and it was conducted considering ethical responsibilities according to the World Medical Association and the Declaration of Helsinki.

\section{Conflict of Interest Statement}

The authors declare that they have no conflicts of interest. 


\section{Funding Sources}

This research did not receive any specific grant from funding agencies in the public, commercial, or not-for-profit sectors.

\section{Author Contributions}

N.D.T.: principal investigator, study conception and design; N.D.T., N.E.: sample collection and molecular analysis; N.D.T.: patient management and interpretation of data. Both authors were involved in drafting the article and/or revising it critically for important intellectual content, and both authors approved the final version to be published.

\section{References}

1 Ruiz de Sabando A, Urrutia Lafuente E, GarcíaAmigot F, Alonso Sánchez A, Morales Garofalo L, Moreno S, et al. Genetic and clinical characterization of BRCA-associated hereditary breast and ovarian cancer in Navarra (Spain) [Erratum in: BMC Cancer. 2019 Dec;19(1): 1227]. BMC Cancer. 2019 Nov;19(1):1145.

2 Harbeck N, Penault-Llorca F, Cortes J, Gnant M, Houssami N, Poortmans P, et al. Breast cancer. Nat Rev Dis Primers. 2019 Sep;5(1): 66.

3 Crawford B, Adams SB, Sittler T, van den Akker J, Chan S, Leitner O, et al. Multi-gene panel testing for hereditary cancer predisposition in unsolved high-risk breast and ovarian cancer patients. Breast Cancer Res Treat. 2017 Jun;163(2):383-90.

4 Sokolenko AP, Bogdanova N, Kluzniak W, Preobrazhenskaya EV, Kuligina ES, Iyevleva AG, et al. Double heterozygotes among breast cancer patients analyzed for BRCA1, CHEK2, ATM, NBN/NBS1, and BLM germ-line mutations. Breast Cancer Res Treat. 2014 Jun; 145(2):553-62.

5 Nomizu T, Matsuzaki M, Katagata N, Kobayashi Y, Sakuma T, Monma T, et al. A case of familial breast cancer with double heterozygosity for BRCA1 and BRCA2 genes. Breast Cancer. 2015 Sep;22(5):557-61.

6 Desai S, Ben-Josef E, Griffith KA, Simeone D, Greenson JK, Francis IR, et al. Gemcitabinebased combination chemotherapy followed by radiation with capecitabine as adjuvant therapy for resected pancreas cancer. Int J Radiat Oncol Biol Phys. 2009 Dec;75(5):1450-5.

7 Tsaousis GN, Papadopoulou E, Apessos A, Agiannitopoulos K, Pepe G, Kampouri S, et al. Analysis of hereditary cancer syndromes by using a panel of genes: novel and multiple pathogenic mutations. BMC Cancer. 2019 Jun;19(1):535.

8 Laitman Y, Friebel TM, Yannoukakos D, Fostira F, Konstantopoulou I, Figlioli G, et al. The spectrum of BRCA1 and BRCA2 pathogenic sequence variants in Middle Eastern, North African, and South European countries. Hum Mutat. 2019 Nov;40(11):e1-23.

9 Podralska MJ, Stembalska A, Ślęzak R, Lewandowicz-Uszyńska A, Pietrucha B, Kołtan S, et al. Ten new ATM alterations in Polish patients with ataxia-telangiectasia. Mol Genet Genomic Med. 2014 Nov;2(6):504-11.

10 Huang Y, Yang L, Wang J, Yang F, Xiao Y, Xia $\mathrm{R}$, et al. Twelve novel Atm mutations identified in Chinese ataxia telangiectasia patients [Erratum in: Neuromolecular Med. 2014 Mar;16] [1] [:216. PMID: 23807571; PMCID: PMC3732755]. Neuromolecular Med. 2013 Sep;15(3):536-40.

11 Beck AC, Yuan H, Liao J, Imperiale P, Shipley K, Erdahl LM, et al. Rate of BRCA mutation in patients tested under NCCN genetic testing criteria. Am J Surg. 2020 Jan;219(1):145-9.

12 Petrucelli N, Daly MB, Pal T. BRCA1- and BRCA2-Associated Hereditary Breast and Ovarian Cancer. In: Adam MP, Ardinger HH, Pagon RA, et al., editors. GeneReviews ${ }^{\circledR}$ [Internet] Seattle: University of Washington, Seattle; 1998 Sep 4. pp. 1993-2020. [Updated 2016 Dec 15]. Available from: https://www. ncbi.nlm.nih.gov/books/NBK1247/

13 Streff H, Profato J, Ye Y, Nebgen D, Peterson SK, Singletary C, et al. Cancer Incidence in First- and Second-Degree Relatives of BRCA1 and BRCA2 Mutation Carriers. Oncologist. 2016 Jul;21(7):869-74.

14 Mersch J, Jackson MA, Park M, Nebgen D, Peterson SK, Singletary C, et al. Cancers associated with BRCA1 and BRCA2 mutations other than breast and ovarian. Cancer. 2015 Jan;121(2):269-75.

15 Swift M, Morrell D, Massey RB, Chase CL. Incidence of cancer in 161 families affected by ataxia-telangiectasia. N Engl J Med. 1991 Dec; 325(26):1831-6.

16 Jerzak KJ, Mancuso T, Eisen A. Ataxia-telangiectasia gene (ATM) mutation heterozygosity in breast cancer: a narrative review. Curr Oncol. 2018 Apr;25(2):e176-80.

17 Royba E, Miyamoto T, Natsuko Akutsu S, Hosoba K, Tauchi H, Kudo Y, et al. Evaluation of ATM heterozygous mutations underlying individual differences in radiosensitivity using genome editing in human cultured cells. Sci Rep. 2017 Jul;7(1):5996.

18 Mullins BT, Gupta G. Increased radiation toxicity with germline ATM variant of uncertain clinical significance. Rep Pract Oncol Radiother. 2019 Nov-Dec;24(6):672-80.

19 Varghese S, Schmidt-Ullrich RK, Dritschilo A, Jung M. Enhanced radiation late effects and cellular radiation sensitivity in an ATM heterozygous breast cancer patient. Radiat Oncol Investig. 1999;7(4):231-7.

20 Broeks A, Braaf LM, Huseinovic A, Nooijen A, Urbanus J, Hogervorst FB, et al. Identification of women with an increased risk of developing radiation-induced breast cancer: a case only study. Breast Cancer Res. 2007;9(2):R26.

21 Dosani M, Schrader KA, Nichol A, Sun S, Shenkier T, Lohn Z, et al. Severe Late Toxicity After Adjuvant Breast Radiotherapy in a Patient with a Germline Ataxia Telangiectasia Mutated Gene: Future Treatment Decisions. Cureus. 2017 Jul;9(7):e1458.

22 Bremer M, Klöpper K, Yamini P, BendixWaltes R, Dörk T, Karstens JH. Clinical radiosensitivity in breast cancer patients carrying pathogenic ATM gene mutations: no observation of increased radiation-induced acute or late effects. Radiother Oncol. 2003 Nov; 69(2):155-60.
23 Nichols KE, Levitz S, Shannon KE, Wahrer DC, Bell DW, Chang G, et al. Heterozygous germline ATM mutations do not contribute to radiation-associated malignancies after Hodgkin's disease. J Clin Oncol. 1999 Apr; 17(4):1259.

24 Oppitz U, Bernthaler U, Schindler D, Sobeck A, Hoehn H, Platzer M, et al. Sequence analysis of the ATM gene in 20 patients with RTOG grade 3 or 4 acute and/or late tissue radiation side effects. Int J Radiat Oncol Biol Phys. 1999 Jul;44(5):981-8.

25 Hasmats J, Kupershmidt I, Rodríguez-Antona C, Su QJ, Khan MS, Jara C, et al. Identification of candidate SNPs for drug induced toxicity from differentially expressed genes in associated tissues. Gene. 2012 Sep;506(1):62-8.

26 Ancot F, Arcand SL, Mes-Masson AM, Provencher DM, Tonin PN. Double PALB2 and BRCA1/BRCA2 mutation carriers are rare in breast cancer and breast-ovarian cancer syndrome families from the French Canadian founder population. Oncol Lett. 2015 Jun;9(6):2787-90.

27 Palmirotta R, Lovero D, Stucci LS, Silvestris E, Quaresmini D, Cardascia A, et al. Double Heterozygosity for BRCA1 Pathogenic Variant and BRCA2 Polymorphic Stop Codon K3326X: A Case Report in a Southern Italian Family. Int J Mol Sci. 2018 Jan;19(1):285.

28 Heidemann S, Fischer C, Engel C, Fischer B, Harder L, Schlegelberger B, et al. Double heterozygosity for mutations in BRCA1 and BRCA2 in German breast cancer patients: implications on test strategies and clinical management. Breast Cancer Res Treat. 2012 Aug; 134(3):1229-39.

29 Andrés R, Menao S, Arruebo M, Quílez E, Cardiel MJ. Double heterozygous mutation in the BRCA1 and ATM genes involved in development of primary metachronous tumours: a case report. Breast Cancer Res Treat. 2019 Oct;177(3):767-70.

30 Bowen TJ, Yakushiji H, Montagna C, Jain S, Ried T, Wynshaw-Boris A. Atm heterozygosity cooperates with loss of Brcal to increase the severity of mammary gland cancer and reduce ductal branching. Cancer Res. 2005 Oct; 65(19):8736-46.

31 Su F, Smilenov LB, Ludwig T, Zhou L, Zhu J, Zhou G, et al. Hemizygosity for Atm and Brcal influence the balance between cell transformation and apoptosis. Radiat Oncol. 2010 Feb;5(1):15-23.

32 Thompson D, Duedal S, Kirner J, McGuffog L, Last J, Reiman A, et al. Cancer risks and mortality in heterozygous ATM mutation carriers. J Natl Cancer Inst. 2005 Jun;97(11):813-22.

33 Choi M, Kipps T, Kurzrock R. ATM Mutations in Cancer: therapeutic Implications. Mol Cancer Ther. 2016 Aug;15(8):1781-91. 